\title{
The Index of Esophageal Cancer Related Ischemic Stroke: A Retrospective Patient Control Study
}

\author{
Yayuan Liu $\mathbb{D}^{1, *}$, Lizhi Lu $\mathbb{D}^{1, *}$, Xuemin Cheng $\mathbb{D}^{\prime}$, Qixiong Qin $\mathbb{D}^{\prime}$, Yunfei Wei $\mathbb{D}^{2}$, Dacheng Wang $\mathbb{D}^{3}$, \\ Haihua Li $\mathbb{D}^{4}$, Guohui Li $\mathbb{D}^{5}$, Hongbin Liang $\mathbb{D}^{6}$, Shengyu Li $\mathbb{D}^{7}$, Zhijian Liang $\mathbb{D}^{1}$ \\ 'Department of Neurology, The First Affiliated Hospital of Guangxi Medical University, Nanning, 53002I, Guangxi Province, People's Republic of \\ China; '2Department of Neurology, The Second Affiliated Hospital of Guangxi Medical University, Nanning, 530007, Guangxi Province, People's \\ Republic of China; ${ }^{3}$ Department of Neurology, The Ninth Affiliated Hospital of Guangxi Medical University, Beihai, 536000, Guangxi Province, \\ People's Republic of China; ${ }^{4}$ Department of Neurology, Fusui County People's Hospital, Chongzuo, 532100, Guangxi Province, People's Republic of \\ China; ${ }^{5}$ Department of Neurology, Wuzhou Red Cross Hospital, Wuzhou, 543002, Guangxi Province, People's Republic of China; ${ }^{6}$ Department of \\ Neurology, Cenxi People's Hospital, Cenxi, 543200, Guangxi Province, People's Republic of China; ${ }^{7}$ Department of Neurology, The Affiliated Wuming \\ Hospital of Guangxi Medical University, Nanning, 530100, Guangxi Province, People's Republic of China
}

*These authors contributed equally to this work

Correspondence: Zhijian Liang, Department of Neurology, First Affiliated Hospital of Guangxi Medical University, 6 Shuangyong Road, Nanning, Guangxi, 530000, People's Republic of China, Tel +86-77I-5330705, Fax +86-77I-5352627, Email liangzhijian@gxmu.edu.cn

Purpose: To investigate independent risk factors for esophageal cancer-related ischemic stroke (ECIS) and to use them to develop an index of ECIS to help clinicians identify patients at high risk for ECIS or to identify ECIS from other types of ischemic stroke.

Methods: We retrospectively enrolled active esophageal cancer (EC) patients with acute ischemic stroke (ECIS group) and patients with active EC without ischemic stroke (EC group), age- and sex-matched with ECIS patients, at seven centers from January 2011 to December 2020. Clinical data and laboratory and imaging findings were collected. Univariate and multivariate analyses were performed to analyze the independent risk factors for ECIS. Optimal cutoffs for sensitivities and specificities were obtained by Youden's J statistic following a receiver operator characteristic (ROC) analysis of each risk factor and the product of the risk factors. Results: A total of 91 ECIS patients and 91 EC patients were included. Elevated levels of carcinoembryonic antigen (CEA) [odds ratio $(\mathrm{OR})=0.105,95 \%$ confidence interval $(\mathrm{CI}): 1.051-1.174, \mathrm{P}<0.001]$, D-dimer $(\mathrm{DD})(\mathrm{OR}=0.003,95 \% \mathrm{CI}: 1.002-1.004, \mathrm{P}<$ $0.001)$, and neutrophil count $(\mathrm{OR}=0.857,95 \% \mathrm{CI}: 1.628-3.407, \mathrm{P}<0.001)$ were independent risk factors for ECIS. The area under the curve (AUC) of each independent risk factor and the product of the three independent risk factors were calculated by a receiver operator characteristic (ROC) curve, and the cutoff value from the largest AUC was called the ECIS index.

Conclusion: It was suggested that elevated plasma DD and CEA levels and increased neutrophils in EC patients may altogether contribute to the development of ECIS. The index of ECIS may facilitate clinicians to identify patients at high risk for ECIS or to identify ECIS from other etiologic types of ischemic stroke.

Keywords: independent risk factors, carcinoembryonic antigen, D-dimer, neutrophil count

\section{Introduction}

Recently, cancer-related ischemic stroke is getting increasing attention. ${ }^{1-3}$ The exact prevalence of cancer-related ischemic stroke remained unknown. However, it was reported that $10 \%$ of patients with ischemic stroke of all causes have known cancer. ${ }^{4}$ And the estimated proportion of embolic stroke of undetermined source patients with cancer similarly approaches $10 \%,{ }^{5}$ although it may be as high as $20 \%$ in some Asian populations. ${ }^{6}$ Previous research has showed that cancer-related ischemic stroke was different from conventional ischemic stroke since cancer-related ischemic stroke was characterized by significantly elevated plasma D-dimer (DD), elevated C-reactive protein, and multiple infarcts distributed in multiple arterial territories. ${ }^{7-10}$ In addition, previous researchers found that the thrombus components of cancer-related ischemic stroke had lower erythrocyte and higher platelet fractions than conventional ischemic stroke, further indicating the difference between cancer-related ischemic stroke and conventional ischemic stroke. ${ }^{11}$ Moreover, our previous studies have found that every confirmed cancer-related ischemic stroke 
has its own unique clinical characteristics and risk factors, suggesting that targeting a certain type of cancer-related ischemic stroke will be more conducive to discovering the clinical characteristics and pathogenesis of cancer-related ischemic stroke. ${ }^{12-14}$ For example, colorectal cancer-related ischemic stroke was associated with elevated CEA and neutrophils, ${ }^{12}$ kidney cancerrelated stroke was associated with elevated plasma D-dimer, CA125 and CEA levels, and increased urine protein levels, ${ }^{13}$ and breast cancer-related ischemic stroke was associated with endocrine therapy, elevated plasma D-dimer and CA153 levels. ${ }^{14}$

Esophageal cancer (EC) is one of the most common digestive tract tumors worldwide. ${ }^{15}$ Remarkably, the incidence of ischemic stroke in patients with EC is 1.5 times that in the normal population, ${ }^{1}$ suggesting that the occurrence of ischemic stroke in some patients was related to EC, and ischemic stroke related to EC is called EC-related ischemic stroke (ECIS). With the development of EC treatment, the survival time of patients with EC has been progressively prolonged, indicating that the number of patients with ECIS will increase. However, the clinical features of ECIS and its independent risk factors are not fully understood, and how to preferably identify ECIS from other etiological types of ischemic stroke is still unclear. In the present study, clinical data for ECIS patients were collected retrospectively to compare with the data from age- and sex-matched control EC patients to explore the independent risk factors for ECIS and to use these risk factors to develop a method to help clinicians identify patients at a high risk for ECIS. This study will contribute to the prevention and treatment of cancer-related ischemic stroke in the future.

\section{Methods}

\section{Patient Selection}

This was a retrospective study of patients with ECIS from January 2011 to December 2020 at The First Affiliated Hospital of Guangxi Medical University, The Second Affiliated Hospital of Guangxi Medical University, The Ninth Affiliated Hospital of Guangxi Medical University, Fusui County People's Hospital, Wuzhou Red Cross Hospital, Cenxi People's Hospital and The Wuming Hospital of Guangxi Medical University. Based on a previous definition of cancer-related ischemic stroke, ${ }^{12-14}$ we defined ECIS as acute ischemic stroke in patients with active EC and without conventional stroke risk factors. In the present study, we defined the conventional stroke risk factors as the presence of hypertension, diabetes, hyperlipidemia, atrial fibrillation, valvular heart disease, and cerebral artery stenosis. The diagnosis of acute ischemic stroke was based on the American Heart Association diagnostic criteria for stroke. ${ }^{16}$ Acute ischemic stroke were confirmed as patients with sudden onset of focal neurological deficits, such as speech blurred, weakness in one or one limb, numbness, and aphasia, who had newly developed ischemic lesions on head CT or MRI that explained the patient's symptoms or sign when hospitalization for active esophageal cancer patients, or for acute ischemic stroke. The ECIS group was enrolled by one oncology expert, one neurology expert, and one neuroimaging expert who were blinded to the study. Patients with primary or metastatic brain cancer or hematological malignancies were excluded because these patients were considered to represent a subgroup with different underlying stroke mechanisms. Patients with cerebral hemorrhage or other cerebrovascular diseases and those with incomplete clinical data were also excluded. Patients with active EC but without IS who were admitted to the same hospital during the same period and who were age- and sex-matched with the ECIS patients were enrolled as the control group. The exclusion criteria included the presence of conventional stroke risk factors, brain metastasis, and other primary cancers (Figure 1). This study was conducted in accordance with the Declaration of Helsinki and approved by the First Affiliated Hospital of Guangxi Medical University Ethical Review Committee. Approval Number: 2021 (KY-E-213). The written informed consent was waivered because of the retrospective nature of our study. During the data collection, personally identifiable information such as names, addresses and phone numbers of the study participants were never recorded. The collected data were kept confidential and used only for the purpose of the study.

\section{Collection of Clinical Data}

We collected general demographic and laboratory data [including blood biochemical tests, coagulation function and DD, carbohydrate antigen (CA) 19-9, and carcinoembryonic antigen (CEA)] from the above two groups of patients. Data including the presence of hypertension, diabetes related to ischemic stroke and other common risk factors for stroke, such as hyperlipidemia, atrial fibrillation, valvular heart disease, and cerebral artery stenosis, were also collected. The examinations for ischemic stroke included cranial computed tomography (CT), magnetic resonance imaging [MRI, including T1-weighted imaging, T2-weighted imaging, fluid-attenuated inversion recovery, and diffusion weighted imaging (DWI)], and the examination of cerebrovascular structural and functional assessments (including cranial color 


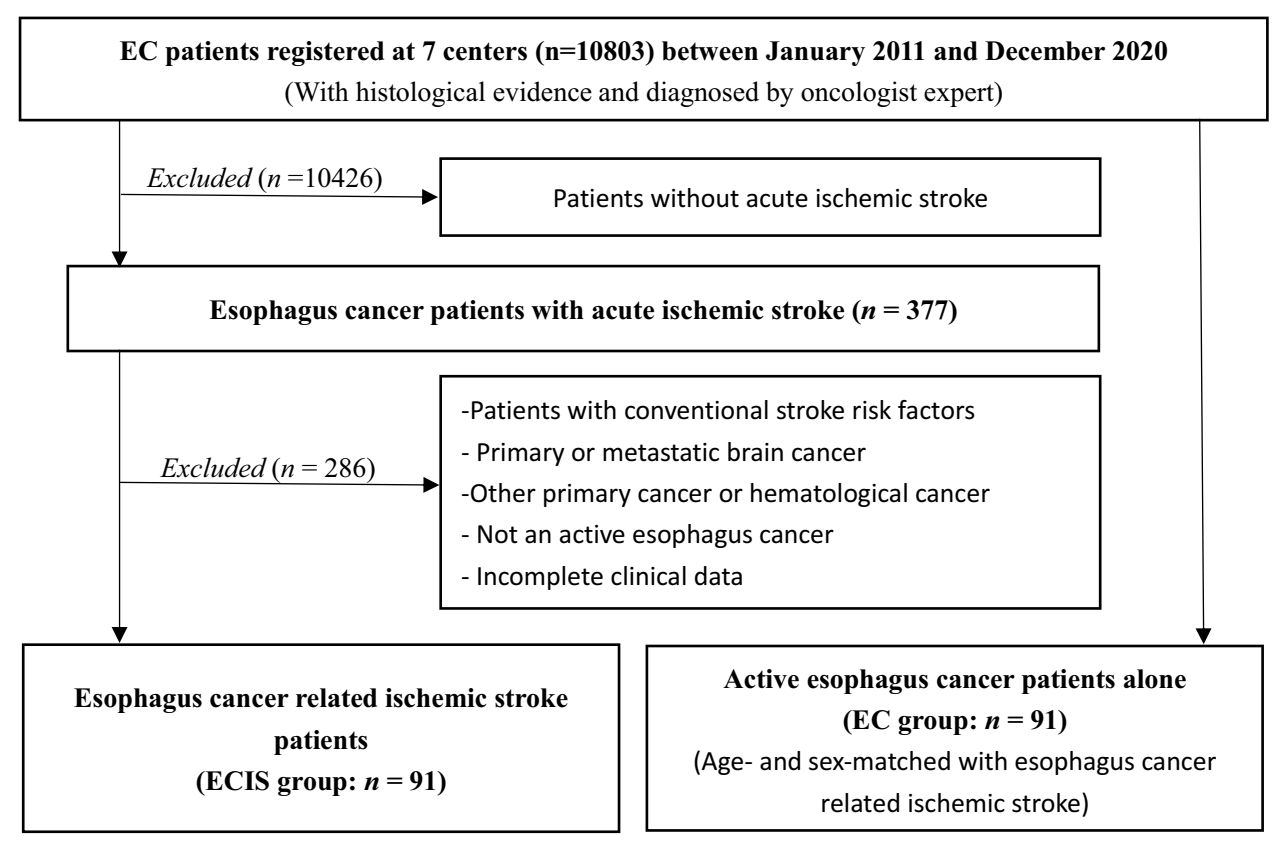

Figure I Patient selection.

Abbreviations: EC, esophagus cancer; ECIS, esophagus cancer related ischemic stroke.

Doppler, magnetic resonance angiography, CT angiography and whole brain digital subtraction angiography). We collected EC-related data, including the clinical manifestations of EC, the pathological types, whether distant metastasis occurred, and the treatment (such as surgery, radiotherapy, chemotherapy, and combination therapy).

\section{Statistical Analysis}

All statistical analyses were performed using SPSS version 24.0 (IBM, Armonk, NY, USA). The independent samples $t$ test was used to compare the continuous variables between the groups, and categorical variables were compared by Pearson's $\chi^{2}$ test or Fisher's exact test. A multivariate logistic regression analysis was performed to identify the independent risk factors for IS in EC patients. Variables with $\mathrm{P}<0.05$ in the univariate analyses were considered explanatory variables and were entered into the multivariate models. To explore the specific biomarkers of ECIS, the ECIS Index was calculated as follows. First, the area under the curve (AUC) of each independent risk and the product of the independent risk factors were calculated by a receiver operator characteristic (ROC) curve; second, the cutoff value from the largest AUC among each risk factor and the product of independent risk factors was called the ECIS Index. All $\mathrm{P}$-values were two-sided, and $\mathrm{P}<0.05$ was considered statistically significant.

\section{Results}

We enrolled 91 ECIS patients, accounting for $0.84 \%$ of all the EC patients. There were $65(71.43 \%)$ male and 26 (28.57\%) female patients, and the patients had a mean age of $63.63 \pm 9.08$ years. As expected, there were no marked differences in age or sex between the two groups. Generally, most patients had squamous cell carcinoma, and others had adenocarcinoma. In addition, 31 (34.07\%) patients had metastases. On the day of ischemic stroke onset, the National Institutes of Health Stroke Scale (NIHSS) score ranged from 0 to 21, and 63 (68.13\%) patients had multiple lesions in multiple vascular areas. The imaging features of the ECIS group, the NIHSS score, and the time interval between the cancer diagnosis and the onset of ischemic stroke are shown in Figure 2 and Table 1. In the ECIS group, most patients experienced ischemic stroke after the EC diagnosis, with 58 (63.74\%) patients experiencing stroke episodes within the first 6 months after the EC diagnosis and nine (9.89\%) within 6 months to 1 year after the EC diagnosis. In addition, 15 (16.48\%) patients had ischemic stroke as the first symptom of occult EC. (Table 1 and Figure 2). 

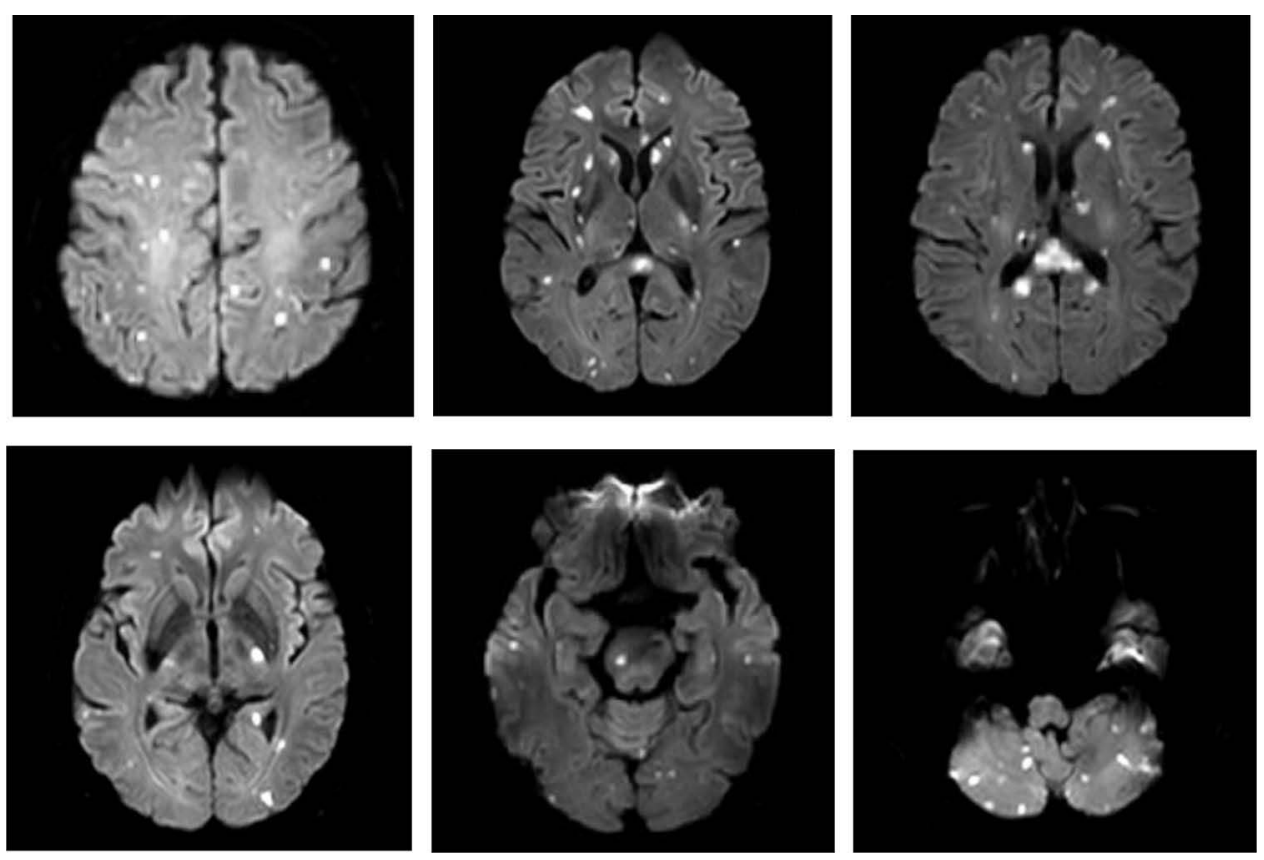

Figure 2 Typical presentation of ECIS. A 50-year-old male patient experienced acute ischemic stroke in the first 2 weeks after diagnosis of EC. DWI showed high signal lesions in the anterior cerebral, middle cerebral, posterior cerebral, anterior and posterior inferior cerebellar arteries territories. CT angiography and echocardiography results were normal.

Compared to the EC group, the neutrophil count, the platelet count, and the DD and CEA levels were higher in the ECIS group, and APTT was shorter $(P<0.05)$. Moreover, there were no significant differences between the two groups regarding treatment, systemic metastasis or the pathological type of EC (Table 2). To identify risk factors for ischemic stroke in EC patients, a multiple logistic regression analysis was performed on the above differences. The results showed that the DD level [odds ratio $(\mathrm{OR})=0.003,95 \%$ confidence interval $(\mathrm{CI}): 1.002-1.004, P<0.001$ ], CEA level (OR $=0.105,95 \%$ CI: $1.051-1.174, P<0.001)$ and neutrophil count (OR $=0.857,95 \%$ CI: $1.628-3.407, P<0.001)$ were independent risk factors for ECIS (Table 3$)$. As in the univariate model of logistic regression, the DD level $(P<0.001)$, CEA level $(P<0.001)$ and neutrophil count $(P<0.001)$ were significantly related to ECIS. The product of three risk factors could be described using the following equation:

Table I Data for Ischemic Stroke Onset, n (\%)

\begin{tabular}{|l|l|}
\hline Characteristics & ECIS (n = 9I) \\
\hline Ischemic stroke lesion pattern & \\
Single arterial territories & $28(31.87 \%)$ \\
Multiple arterial territories & $63(68.13 \%)$ \\
NIHSS scores on day of ischemic stroke onset & \\
$0-4$ & $35(38.46 \%)$ \\
$5-15$ & $43(47.25 \%)$ \\
I5-20 & $11(12.08 \%)$ \\
$>20$ & $2(2.20 \%)$ \\
Time interval between diagnosis of EC and stroke & $15(16.48 \%)$ \\
Ischemic stroke as the first manifestation of ECIS & $58(63.74 \%)$ \\
Ischemic stroke onset after ECIS diagnosis & $9(9.89 \%)$ \\
$0-6$ months & $9(9.89 \%)$ \\
$6-12$ months & \\
$>$ I year &
\end{tabular}

Abbreviations: ECIS, esophageal cancer related ischemic stroke; NIHSS, National Institutes of Health Stroke Scale. 
Table 2 Clinical Characteristic of ECIS Compared to EC

\begin{tabular}{|c|c|c|c|}
\hline Characteristics & ECIS $(n=91)$ & $E C(n=91)$ & $P$ \\
\hline Age & $63.63 \pm 9.08$ & $63.66 \pm 7.30$ & $0.98^{\mathrm{a}}$ \\
\hline \multicolumn{4}{|l|}{ Gender } \\
\hline Male, n (\%) & $65(71.43)$ & $65(71.43)$ & $1.00^{\mathrm{b}}$ \\
\hline Female, n (\%) & $26(28.57)$ & $26(28.57)$ & $1.00^{\mathrm{b}}$ \\
\hline \multicolumn{4}{|l|}{ Blood tests } \\
\hline WBC $\left(10^{9} / \mathrm{L}\right)$ & $7.05 \pm 2.09$ & $6.69 \pm 1.64$ & $0.20^{\mathrm{a}}$ \\
\hline $\operatorname{RBC}\left(10^{9} / \mathrm{L}\right)$ & $4.21 \pm 0.57$ & $4.23 \pm 0.52$ & $0.85^{\mathrm{a}}$ \\
\hline $\mathrm{HGB}(\mathrm{g} / \mathrm{L})$ & $120.20 \pm 19.83$ & $125.16 \pm 16.41$ & $0.07^{\mathrm{a}}$ \\
\hline Platelet $\left(10^{9} / \mathrm{L}\right)$ & $271.13 \pm 87.04$ & $243.94 \pm 79.53$ & $0.03^{\mathrm{a}}$ \\
\hline Neutrophil count $\left(10^{9} / \mathrm{L}\right)$ & $5.33 \pm 1.55$ & $4.32 \pm 1.34$ & $<0.01^{\mathrm{a}}$ \\
\hline Lymphocyte count $\left(10^{9} / \mathrm{L}\right)$ & $1.69 \pm 0.82$ & $1.76 \pm 0.59$ & $0.53^{\mathrm{a}}$ \\
\hline PT (s) & $11.44 \pm 1.52$ & $11.32 \pm 1.09$ & $0.57^{\mathrm{a}}$ \\
\hline INR & $0.98 \pm 0.12$ & $0.97 \pm 0.10$ & $0.50^{\mathrm{a}}$ \\
\hline APTT (s) & $30.45 \pm 2.61$ & $31.28 \pm 1.91$ & $0.02^{\mathrm{a}}$ \\
\hline FIB $(g / L)$ & $4.49 \pm 1.63$ & $4.73 \pm 1.19$ & $0.26^{\mathrm{a}}$ \\
\hline D-dimer (ng/mL) & $|484.76 \pm| 424.6 \mid$ & $500.53 \pm 276.56$ & $<0.01^{\mathrm{a}}$ \\
\hline CEA $(\mathrm{ng} / \mathrm{mL})$ & $|3.37 \pm 9.4|$ & $8.41 \pm 5.94$ & $<0.01^{\mathrm{a}}$ \\
\hline CAI $25(\mathrm{U} / \mathrm{mL})$ & $91.86 \pm 102.99$ & $72.91 \pm 83.18$ & $0.17^{\mathrm{a}}$ \\
\hline CAI99 (U/mL) & $88.79 \pm 179.38$ & $52.80 \pm 57.09$ & $0.07^{\mathrm{a}}$ \\
\hline Type of EC & & & $0.64^{\mathrm{b}}$ \\
\hline Squamous cell carcinomas & $80(87.91)$ & $82(90.11)$ & \\
\hline Adenocarcinoma & II (12.09) & $9(9.89)$ & \\
\hline Systemic metastasis, n (\%) & & & $0.10^{\mathrm{b}}$ \\
\hline Yes & 31 (34.07) & $21(23.08)$ & \\
\hline No & $60(65.93)$ & $70(76.92)$ & \\
\hline \multicolumn{4}{|l|}{ Methods of therapy, $n(\%)$} \\
\hline Surgery & $33(36.26)$ & $45(49.45)$ & $0.07^{\mathrm{b}}$ \\
\hline Chemotherapy & $19(20.88)$ & $30(32.97)$ & $0.06^{\mathrm{b}}$ \\
\hline Radiotherapy & $27(29.67)$ & $37(40.66)$ & $0.12^{\mathrm{b}}$ \\
\hline No treatment & $36(39.56)$ & $20(21.98)$ & $0.10^{\mathrm{b}}$ \\
\hline
\end{tabular}

Notes: ${ }^{a}$ With two independent samples $t$ - test; ${ }^{b}$ with $\chi 2$ test.

Abbreviations: WBC, white blood cell; RBC, red blood cell; HGB, hemoglobin; PT, prothrombin time; INR, international normalized ratio; FIB, fibrinogen; CRP, C-reactive protein; CEA, carcinoembryonic antigen; CA, cancer antigen.

$\operatorname{Logist}(p)=\operatorname{In}(p /(1-p))=\beta_{0}+\beta_{1} \mathrm{X}_{1}+\beta_{2} \mathrm{X}_{2}+\beta_{3} \mathrm{X}_{3}$, where $p$ is probability of ischemic stroke, $\mathrm{X} 1$ is the DD level, $\mathrm{X} 2$ is the CEA level, and $\mathrm{X} 3$ is the neutrophil count.

The ROC regression analysis showed that among each risk factor, the area under the curve (AUC) for the product of all three risk factors was the largest at $0.887 \pm 0.025$ (95\% CI: $0.838-0.936, P<0.001)$, with a sensitivity of $72.5 \%$ and a specificity of $94.5 \%$. The cutoff value of the product of all three risk factors equaled 1518.22 , which was called the index of ECIS in this study (Table 4 and Figure 3).

Table 3 Multivariate Logistic Regression Analysis

\begin{tabular}{|l|l|l|l|l|l|l|l|}
\hline Factors & $\boldsymbol{\beta}$ & SE & WALs & df & $\boldsymbol{P}$ & OR & $\mathbf{9 5 \%} \mathbf{C I}$ \\
\hline D-dimer & 0.003 & 0.001 & 24.382 & $I$ & $<0.00 \mathrm{I}$ & 1.001 & $1.002-1.004$ \\
CEA & 0.105 & 0.028 & 13.752 & $\mathrm{I}$ & $<0.00 \mathrm{I}$ & $\mathrm{I} .067$ & $1.05 \mathrm{I}-\mathrm{I} .174$ \\
Neutrophil count & 0.857 & 0.188 & 20.684 & $\mathrm{I}$ & $<0.00 \mathrm{I}$ & 1.945 & $1.628-3.407$ \\
Constant & -4.653 & 3.054 & $2.23 \mathrm{I}$ & $\mathrm{I}$ & 0.128 & 0.010 & \\
\hline
\end{tabular}

Abbreviations: $\mathrm{CEA}$, carcinoembryonic antigen; $\mathrm{SE}$, standard error; $\mathrm{OR}$, odds ratio; $\mathrm{Cl}$, confidence interval. 
Table 4 Results of ROC Analyses

\begin{tabular}{|l|l|l|l|l|l|l|}
\hline Variable & AUC & SE & Sensitivity & Specificity & Youden Index & $\mathbf{9 5 \%}$ CI \\
\hline Product of three risk factors & 0.887 & 0.025 & 0.725 & 0.945 & 0.670 & $0.838-0.936$ \\
D-dimer & 0.772 & 0.035 & 0.538 & 0.945 & 0.483 & $0.704-0.841$ \\
CEA & 0.645 & 0.041 & 0.473 & 0.844 & 0.319 & $0.564-0.726$ \\
Neutrophil count & 0.746 & 0.036 & 0.670 & 0.736 & 0.406 & $0.675-0.817$ \\
\hline
\end{tabular}

Abbreviations: AUC, area under curve; $\mathrm{Cl}$, confidence interval; CEA, carcinoembryonic antigen.

\section{Discussion}

Cancer-related ischemic stroke has received increasing attention, and some clinical features of cancer-related ischemic stroke have been found. First, previous studies have demonstrated that cancer-related ischemia may occur after or before the diagnosis of cancer. ${ }^{12-14}$ In the present study, a similar finding was also revealed in patients with ECIS, indicating that some measures should be taken to prevent ischemic stroke when the diagnosis of EC is obtained or when diagnosing the etiology of a cryptogenic ischemic stroke EC. Second, previous studies found that the clinical features of cancer-related ischemic stroke included elevated plasma DD levels and multiple ischemic lesions distributed in different cerebral arterial territories. ${ }^{8,10,17}$ In the present study, the ECIS patients had similar features, which indicated that ECIS shared the common clinical features of cancer-related ischemic stroke. However, in the present study, compared with the EC group, elevated CEA levels and increased neutrophil and platelet counts were found in the ECIS group, suggesting that ECIS not only had its own features but also has a unique pathogenesis. Moreover, in the present study, elevated DD and CEA levels and neutrophil counts were found to be independent risk factors for ECIS, further suggesting that ECIS has a unique pathogenesis and that these independent risk factors may play a role in the pathogenesis of ECIS.

The pathogenesis of cancer-related ischemic stroke has received attention for years because its pathogenesis may influence the choice of management, stroke prognosis, and risk of recurrence. Previous studies have shown that the pathogenesis of cancer-related ischemic stroke is mainly associated with hypercoagulability and that elevated plasma DD levels ${ }^{18,19}$ are an important biomarker. Moreover, elevated plasma DD levels in cancer patients were found to be associated with the pathogenesis of ischemic stroke through increasing microthrombi in the bilateral common carotid arteries, ${ }^{20}$ In the present study, elevated plasma DD levels in the EC patients may also lead to ECIS through the pathway mentioned above. In addition, in the present study, elevated plasma CEA levels were found. It is not clear what role that CEA plays in the generation of ECIS. However, animal experiments have confirmed that mucins secreted by cancer cells into the blood may

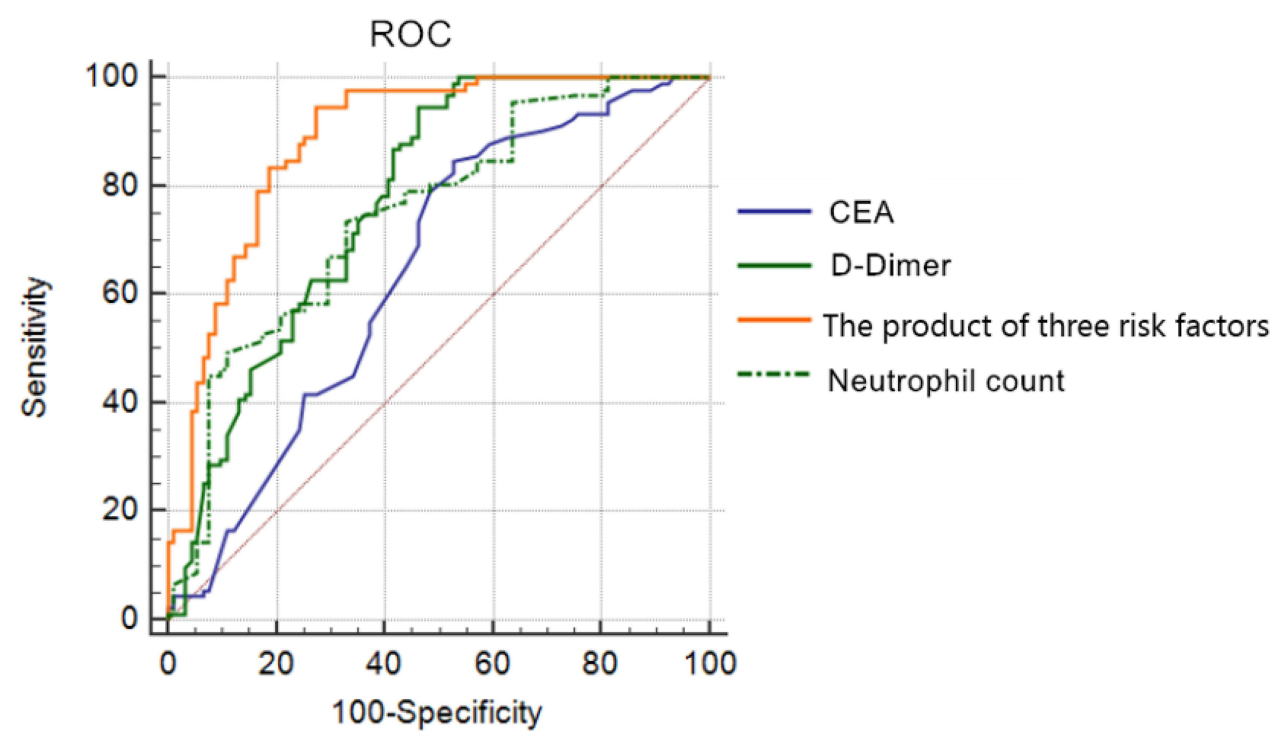

Figure 3 ROC curve analysis of the product of CEA, D-dimer, neutrophil count and the product of all of the three risk factors. 
first bind to platelets with P-selectin, ${ }^{21,22}$ which is an adhesion molecule on the membrane surface of neutrophils, and then the mucins activate platelets and release soluble P-selectin, finally leading to hypercoagulability and thrombosis. ${ }^{23-25}$ Moreover, elevated levels of plasma CA125, another mucin factor that is secreted into the blood by cancer cells, were found to increase blood coagulation and thus lead to cancer-related stroke. ${ }^{26}$ As it is also a mucinous factor that is secreted by EC cells, it was speculated that CEA may be involved in the pathogenesis of ECIS. Since increased neutrophil counts have been found to be associated with the development of cancer-related ischemic stroke, ${ }^{12-14}$ it was suggested that elevated DD and CEA levels and increased neutrophils might play important roles in the development of ECIS. Compared with our previous studies, ${ }^{12-14}$ ECIS and other cancer related ischemic stroke shared similar clinical features, such as ischemic stroke occurring within the first 6 months after the EC diagnosis, multiple ischemic lesions in multiple vascular areas and the pathogenesis of ischemic stroke associating with hypercoagulability. However, ECIS may have unique features including elevated plasma DD and CEA levels accompanied by increased neutrophils.

How to exactly identify a cancer-related ischemic stroke remains a clinical challenge. Previous studies have made a variety of efforts, and these studies have evaluated several factors, such as increased levels of DD, C-reactive protein and neutrophil/lymphocyte ratio in the blood and the presence of multiple ischemic lesions in neuroimaging, which have been used as biomarkers of cancer-related ischemic stroke. ${ }^{27-30}$ However, due to the lack of specific substances or measures, the precise measures to identify cancer-related cerebral infarction are still unclear. In this study, considering that the three independent risk factors for ECIS may play their own roles or may play a role together as a factor in the development of ECIS, we established an ROC curve to identify the factors that play the greatest role in the development of ECIS. As a result, the ROC curve analysis showed that the AUC of the product of the three risk factors was the largest, had a sensitivity of $72.5 \%$, a specificity of $94.5 \%$, and a cutoff value equal to 1518.22 , indicating that the three risk factors play an overall role in the development of ECIS. With the ECIS index, clinicians could identify patients at high risk for ECIS or can more easily identify ECIS patients from EC patients with ischemic stroke. In addition, the concept of ECIS and its clinical characteristics and possible pathogenesis have raised more people's attention not only to prolong the life expectancy of patients with EC, but also to enhance the quality of life of patients with EC through effectively preventive and therapeutic measures for ECIS. ${ }^{31}$

The main limitations of our study included the selection bias caused by the small sample size and some uncontrollable settings in the retrospective study. Nevertheless, we provide a new method to determine or predict ECIS. Further prospective studies with larger sample sizes are needed to verify the role of the ECIS index.

\section{Conclusion}

In summary, our results suggest that elevated plasma DD and CEA levels and increased neutrophils in EC patients may altogether contribute to the development of ECIS. The ECIS index may help clinicians to identify patients at a high risk for ECIS or to identify ECIS from EC patients with ischemic stroke.

\section{Abbreviations}

EC, esophageal cancer; ECIS, esophageal cancer-related ischemic stroke; ROC, receiver operator characteristic; CEA, carcinoembryonic antigen; OR, odds ratio; CI, confidence interval; DD, D-dimer; AUC, area under the curve; CT, computed tomography; MRI, magnetic resonance imaging; DWI, diffusion weighted imaging; NIHSS, National Institutes of Health Stroke Scale; WBC, white blood cell; RBC, red blood cell; HGB, hemoglobin; PT, prothrombin time; INR, international normalized ratio; FIB, fibrinogen; CRP, C-reactive protein; CA, cancer.

\section{Acknowledgments}

We would like to thank Prof. Li for her guidance on statistical methods. We are grateful for the advice and discussions with our teammate Shijian Chen and Gengyu Cen. Yayuan Liu and Lizhi Lu are co-first authors for this study.

\section{Funding}

This study was funded by the National Key R\&D Program of China (No. 2018YFC1311300), the Foundation of Science and Technology Plan Projects of Qingxiu District of Nanning (No. 2020043). 


\section{Disclosure}

The authors of this manuscript declare no relationships and no conflicts with any companies, whose products or services may be related to the subject matter of the article.

\section{References}

1. Zoller B, Ji J, Sundquist J, et al. Risk of haemorrhagic and ischaemic stroke in patients with cancer: a nationwide follow-up study from Sweden. Eur J Cancer. 2012;48:1875-1883. doi:10.1016/j.ejca.2012.01.005

2. Devulapalli S, Pinto N, Gandothra C, et al. A rare case of occipital stroke as a consequence of nonbacterial thrombotic endocarditis in ovarian clear cell carcinoma: a case report. Case Rep Neurol. 2012;4:84-91. doi:10.1159/000338811

3. Kono T, Ohtsuki T, Hosomi N, et al. Cancer-associated ischemic stroke is associated with elevated d-dimer and fibrin degradation product levels in acute ischemic stroke with advanced cancer. Geriatr Gerontol Int. 2012;12:468-474. doi:10.1111/j.1447-0594.2011.00796.x

4. Sanossian N, Djabiras C, Mack WJ, et al. Trends in cancer diagnoses among inpatients hospitalized with stroke. J Stroke Cerebrovasc Dis. 2013;22:1146-1150. doi:10.1016/j.jstrokecerebrovasdis.2012.11.016

5. Ntaios G, Perlepe K, Lambrou D, et al. Prevalence and overlap of potential embolic sources in patients with embolic stroke of undetermined source. $J$ Am Heart Assoc. 2019;8:e012858. doi:10.1161/JAHA.119.012858

6. Kim SJ, Park JH, Lee MJ, et al. Clues to occult cancer in patients with ischemic stroke. PLoS One. 2012;7:e44959. doi:10.1371/journal. pone.0044959

7. Braemswig TB, Usnich T, Albach FN, et al. Early new diffusion-weighted imaging lesions appear more often in stroke patients with a multiple territory lesion pattern. Stroke. 2013;44:2200-2204. doi:10.1161/STROKEAHA.111.000810

8. Kim J, Jung K, Park KH, et al. Clinical manifestation of cancer related stroke: retrospective case-control study. J Neuro-Oncol. 2013;111:295-301. doi:10.1007/s11060-012-1011-4

9. Guo Y, Chang M, Chen P, et al. Predictive value of plasma d-Dimer levels for cancer-related stroke: a 3-Year retrospective study. $J$ Stroke and Cerebrovasc Dis. 2014;3:e249-e254. doi:10.1016/j.jstrokecerebrovasdis.2013.10.022

10. Kassubek R, Bullinger L, Kassubek J, et al. Identifying ischemic stroke associated with cancer: a multiple model derived from a case-control analysis. J Neurol. 2017;264:781-791. doi:10.1007/s00415-017-8432-0

11. Park H, Kim J, Ha J, et al. Histological features of intracranial thrombi in stroke patients with cancer. Ann Neurol. 2019;86:143-149. doi:10.1002/ ana.25495

12. Qin Q, Cheng X, Lu L, et al. Biomarkers and potential pathogenesis of colorectal cancer-related ischemic stroke. World $J$ Gastroentero. 2018;24:4950-4958. doi:10.3748/wjg.v24.i43.4950

13. Jiang H, Qin C, Cheng D, et al. Potential pathogenesis and biomarkers of kidney Cancer-Related stroke. Med Sci Monitor. 2017;23:2292-2298. doi:10.12659/MSM.904710

14. Cheng X, Qin Q, Lu L, et al. The independent risks and specific biomarker of breast cancer-related ischemic stroke. Int $J$ Neurosci. 2021;131:135-143. doi:10.1080/00207454.2020.1733562

15. Bray F, Ferlay J, Soerjomataram I, et al. Global cancer statistics 2018: GLOBOCAN estimates of incidence and mortality worldwide for 36 cancers in 185 countries. CA Cancer J Clin. 2018;68:394-424. doi:10.3322/caac.21492

16. Powers WJ, Rabinstein AA, Ackerson T, et al. Guidelines for the early management of patients with acute ischemic stroke: 2019 update to the 2018 guidelines for the early management of acute ischemic stroke: a guideline for healthcare professionals from the American Heart Association/ American Stroke Association. Stroke. 2019;50:e344-e418. doi:10.1161/STR.00000000000000211

17. Kim K, Lee J. Risk factors and biomarkers of ischemic stroke in cancer patients. J Stroke. 2014;16:91-96. doi:10.5853/jos.2014.16.2.91

18. Guyatt GH, Akl EA, Crowther M, et al. Introduction to the ninth edition. Chest. 2012;141:48S-52S. doi:10.1378/chest.11-2286

19. Schwarzbach CJ, Schaefer A, Ebert A, et al. Stroke and cancer: the importance of cancer-associated hypercoagulation as a possible stroke etiology. Stroke. 2012;43:3029-3034. doi:10.1161/STROKEAHA.112.658625

20. Seok JM, Kim SG, Kim JW, et al. Coagulopathy and embolic signal in cancer patients with ischemic stroke. Ann Neurol. 2010;68:213-219. doi:10.1002/ana.22050

21. Navi BB, Sherman CP, Genova R, et al. Mechanisms of ischemic stroke in patients with cancer: a Prospective Study. Ann Neurol. 2021;90:159-169. doi:10.1002/ana.26129

22. Shao B, Wahrenbrock MG, Yao L, et al. Carcinoma mucins trigger reciprocal activation of platelets and neutrophils in a murine model of Trousseau syndrome. Blood. 2011;118:4015-4023. doi:10.1182/blood-2011-07-368514

23. Riedl J, Hell L, Kaider A, et al. Association of platelet activation markers with cancer-related venous thromboembolism. Platelets. 2016;27:80-85. doi:10.3109/09537104.2015.1041901

24. Ferroni P, Martini F, Riondino S, et al. Soluble P-selectin as a marker of in vivo platelet activation. Clin Chim Acta. 2009;399:88-91. doi:10.1016/j. cca.2008.09.018

25. Ay C, Simanek R, Vormittag R, et al. High plasma levels of soluble P-selectin are predictive of venous thromboembolism in cancer patients: results from the Vienna Cancer and Thrombosis Study (CATS). Blood. 2008;112:2703-2708. doi:10.1182/blood-2008-02-142422

26. Jovin TG, Boosupalli V, Zivkovic SA, et al. High titers of CA-125 may be related with recurrent ischemic strokes in patients with cancer. Neurology. 2005;64:1944-1945. doi:10.1212/01.WNL.0000163850.07976.63

27. Jiang J, Shang X, Zhao J, et al. Score for predicting active cancer in patients with ischemic stroke: a Retrospective Study. Biomed Res Int. $2021 ; 4: 9$.

28. Kawano T, Sasaki T, Gon Y, et al. High neutrophil/lymphocyte ratio at cancer diagnosis predicts incidence of stroke in cancer patients. Brain Commun. 2021;3:fcab071. doi:10.1093/braincomms/fcab071 
29. Hasegawa Y, Setoguchi T, Sakaida T, et al. Utility of a scoring system for differentiating cancer-associated stroke from cryptogenic stroke in patients with cancer. Neurol Sci. 2020;41(5):1245-1250. doi:10.1007/s10072-019-04231-5

30. Wang JY, Zhang GJ, Zhuo SX, et al. D-dimer $>2.785 \mathrm{mug} / \mathrm{mL}$ and multiple infarcts 3 vascular territories are two characteristics of identifying cancer-associated ischemic stroke patients. Neurol Res. 2018;40:948-954. doi:10.1080/01616412.2018.1504179

31. Chao BH, Yan F, Hua Y, et al. Stroke prevention and control system in China: CSPPC-stroke program. Int J Stroke. 2021;16(3):265-272. doi:10.1177/1747493020913557

\section{Publish your work in this journal}

Neuropsychiatric Disease and Treatment is an international, peer-reviewed journal of clinical therapeutics and pharmacology focusing on concise rapid reporting of clinical or pre-clinical studies on a range of neuropsychiatric and neurological disorders. This journal is indexed on PubMed Central, the 'PsycINFO' database and CAS, and is the official journal of The International Neuropsychiatric Association (INA). The manuscript management system is completely online and includes a very quick and fair peer-review system, which is all easy to use. Visit http://www.dovepress.com/testimonials.php to read real quotes from published authors.

Submit your manuscript here: https://www.dovepress.com/neuropsychiatric-disease-and-treatment-journal 